\title{
Quantifying hyperoxia-mediated damage to mammalian respiratory cilia-driven fluid flow using particle tracking velocimetry optical coherence tomography
}

Ute A. Gamm

Brendan K. Huang

Mansoor Syed

Xuchen Zhang

Vineet Bhandari

Michael A. Choma 


\section{Quantifying \\ hyperoxia-mediated damage to mammalian respiratory cilia-driven fluid flow using particle tracking velocimetry optical coherence tomography}

\author{
Ute A. Gamm, ${ }^{a, *}$ Brendan K. Huang, ${ }^{b}$ Mansoor Syed, ${ }^{c, \dagger}$ \\ Xuchen Zhang, ${ }^{d}$ Vineet Bhandari, ${ }^{c, \neq}$ and \\ Michael A. Choma ${ }^{a, b, c, e, *}$ \\ aYale University, Department of Diagnostic Radiology, P.O. Box \\ 208043, New Haven, Connecticut 06520, United States \\ ${ }^{b}$ Yale University, Department of Biomedical Engineering, 55 Prospect \\ Street, New Haven, Connecticut 06520, United States \\ 'Yale University, Department of Pediatrics, P.O. Box 208064, New \\ Haven, Connecticut 06520, United States \\ ¿Yale University, Department of Pathology, PO Box 208023, New \\ Haven, Connecticut 06520, United States \\ eYale University, Department of Applied Physics, P.O. Box 208267, \\ New Haven, Connecticut 06520, United States
}

\begin{abstract}
Oxygen supplementation [hyperoxia, increased fraction of inspired oxygen $\left(\mathrm{FiO}_{2}\right)$ ] is an indispensable treatment in the intensive care unit for patients in respiratory failure. Like other treatments or drugs, hyperoxia has a riskbenefit profile that guides its clinical use. While hyperoxia is known to damage respiratory epithelium, it is unknown if damage can result in impaired capacity to generate cilia-driven fluid flow. Here, we demonstrate that quantifying cilia-driven fluid flow velocities in the sub- $100 \mu \mathrm{m} / \mathrm{s}$ regime (sub-0.25 in./min regime) reveals hyperoxia-mediated damage to the capacity of ciliated respiratory mucosa to generate directional flow. Flow quantification was performed using particle tracking velocimetry optical coherence tomography (PTV-OCT) in ex vivo mouse trachea. The ability of PTV-OCT to detect biomedically relevant flow perturbations in the sub- $100 \mu \mathrm{m} / \mathrm{s}$ regime was validated by quantifying temperature- and drug-mediated modulation of flow performance in ex vivo mouse trachea. Overall, PTV-OCT imaging of cilia-driven fluid flow in ex vivo mouse trachea is a powerful and straightforward approach for studying factors that modulate and damage mammalian respiratory ciliary physiology. @ The Authors. Published by SPIE under a Creative Commons Attribution 3.0 Unported License. Distribution or reproduction of this work in whole or in part requires full attribution of the original publication, including its DOI. [DOI: 10.1117/1.JBO.20.8.080505]
\end{abstract}

\footnotetext{
*Address all correspondence to: Ute A. Gamm, E-mail: ute.gamm@yale.edu; Michael A. Choma, E-mail: michael.choma@yale.edu

${ }^{\dagger}$ Current affiliation: Drexel University College of Medicine, Philadelphia, Pennsylvania 19129, United States

${ }^{\ddagger}$ Current affiliations: Drexel University College of Medicine, Philadelphia, Pennsylvania 19129, United States and St. Christopher's Hospital for
} Children, Philadelphia, Pennsylvania 19134, United States
Keywords: optical coherence tomography; particle tracking velocimetry optical coherence tomography; particle tracking; velocimetry; physiology; cilia; respiratory; oxygen; hyperoxia.

Paper 150336LR received May 18, 2015; accepted for publication Jul. 27, 2015; published online Aug. 26, 2015.

Oxygen supplementation [hyperoxia, increased fraction of inspired oxygen $\left.\left(\mathrm{FiO}_{2}\right)\right]$ is an indispensable treatment in the intensive care unit for patients in respiratory failure. In respiratory failure, decreased hemoglobin-oxygen saturation leads to decreased rates of end-organ oxygen delivery. Increased $\mathrm{FiO}_{2}$ can increase hemoglobin-oxygen saturation that, in turn, increases the rate of oxygen delivery to end-organs. Supplemental oxygen is widely used in respiratory failure of various etiologies, including prematurity-associated lung disease ${ }^{1,2}$ to asthma $^{3}$ to chronic obstructive lung disease. ${ }^{4}$ However, as with any other therapeutic intervention, hyperoxia has negative side effects. Increased oxygen levels lead to the creation of reactive oxygen species, that causes cellular damage. ${ }^{1,5,6}$ Hyperoxia damages respiratory epithelial cells ${ }^{5,7}$ and may have significant impact on neonatal development. ${ }^{8-11}$ To date, the optimum dosage of oxygen for both adults ${ }^{12,13}$ and neonates ${ }^{14}$ remains a topic of debate. One important aspect of the debate is that, while hyperoxia is known to be generally toxic to cells, the downstream physiological consequences of that toxicity are incompletely understood.

Although it is established that hyperoxia damages respiratory epithelia surfaces, there is limited research into the impact of hyperoxia on the net flow performance of respiratory ciliated surfaces. Since ciliated surfaces are responsible for clearing mucus from the lungs, oxygen-mediated damage to those surfaces may impair mucus clearance, an important line of defense against infection and harmful particulate matter. Very high levels of oxygen can lead to histologically evident changes in respiratory epithelium, such as deciliation, ${ }^{15}$ and increased thickness of epithelium and smooth muscle layer. ${ }^{16,17}$ However, the consequences of those changes on directional flow rates at the $\sim 100$ to $1000 \mu \mathrm{m}$ size scale are not well understood. Sackner et al. ${ }^{18}$ found a dose- and time-dependent inhibition of tracheal mucus flow in live dogs. Tracheal mucus flow rates were quantified through videobronchoscopic imaging of Teflon disk tracers. One potential drawback of an in vivo approach is a limited ability to uncouple the effects of hyperoxia on the performance of ciliated epithelium and the effects of hyperoxia on mucus itself. ${ }^{19}$ Therefore, there are opportunities for optical imaging based flow diagnostics to improve our understanding of the sideeffects profile of hyperoxia, especially as it relates to cilia-driven fluid flow performance.

We previously demonstrated that cilia-driven fluid flow can be quantified using particle tracking velocimetry optical coherence tomography (PTV-OCT). ${ }^{20,21}$ PTV-OCT is able to track particles in both transverse and axial directions. In contrast, traditional Doppler OCT is limited to axial velocimetry, which would fail to capture the predominantly transverse flow component of cilia-driven fluid flow. In previous publications, we demonstrated the feasibility of PTV-OCT ${ }^{20}$ in the $\sim 100$ to $1000 \mu \mathrm{m} / \mathrm{s}$ flow velocity regime and recently demonstrated that the method is sensitive enough to quantify subtle variability in cilia-driven flow performance in Xenopus embryos. ${ }^{21}$ In this 

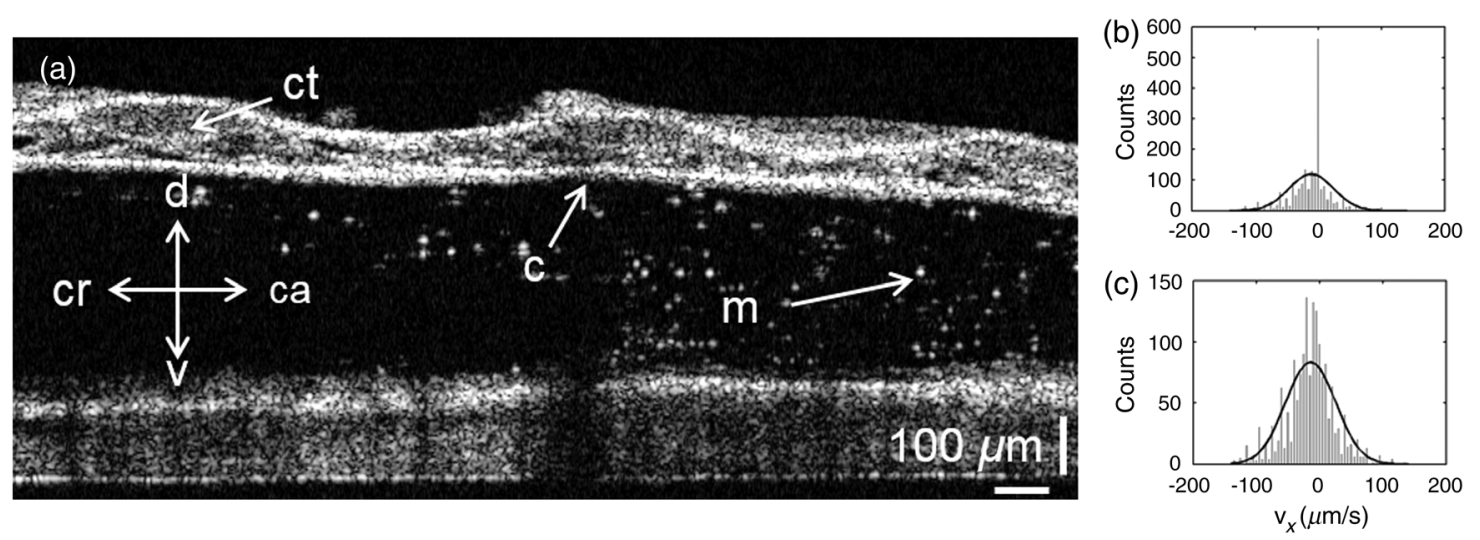

Fig. 1 (a) Cross-sectional image of ex vivo mouse trachea seeded with polystyrene microbeads to visualize cilia-driven fluid flow. C, cilia; m, microspheres; ct, cartilage; cr, cranial; ca, caudal; v, ventral; d, dorsal. (b) and (c) Histograms of flow velocity statistics were used to quantify average flow speed over a region of interest near the ciliated tracheal luminal surface. A larger than expected number of particle velocity counts were typically present in the zero velocity histogram bin (b). These stationary particles were excluded, and average flow speeds were calculated from the resultant histograms (c) (Video 1, MP4, 2.94 MB) [URL: http://dx.doi.org/10.1117/1.JBO.20.8.080505.1].

study, we demonstrate that OCT-PTV is capable of imaging and quantifying cilia-driven fluid flow inside the lumen of ex vivo adolescent mouse tracheae. In order to quantify tracheal ciliadriven fluid flow in the sub- $100 \mu \mathrm{m} / \mathrm{s}$ regime, we refined our processing of PTV-OCT data. As a straightforward demonstration of the utility of PTV-OCT in mouse tracheal ciliary physiology, we quantified the temperature adenosine triphosphate (ATP) dependence of cilia-driven fluid flow. After validating of our approach in this relevant biological context, we next investigated the influence of sustained hyperoxia on the capacity of the tracheal epithelial surface to drive cilia-driven fluid flow. Our quantitative imaging results support the hypothesis that hyperoxia may be a significant modifier of cilia-driven fluid flow and mucus clearance.

Figure 1 and Video 1 show a cross-sectional image and movie, respectively, of an ex vivo adolescent mouse trachea [postnatal day (PND) 21] with $5 \mu \mathrm{m}$ polystyrene particle flow tracers seeded into the lumen. We used a Thorlabs Telesto OCT system $\left(\lambda_{o}=1300 \mathrm{~nm}\right)$. Tracheae were imaged in warmed phosphate-buffered saline (PBS). Prior to imaging, tracheae were flushed with PBS to remove mucus. The dorsal aspect of the tracheae was positioned at the top of the OCT image field of view. Here, the $z$ axis is parallel to the optical axis and the $x$ axis is nominally parallel to the cranial-caudal anatomic axis. In order to generate an estimate of the flow velocity $\mathbf{v}(x, z)=v_{x}(x, z) \mathbf{i}+v_{z}(x, z) \mathbf{j}$, we performed two-dimensional, two-vector component velocimetry using PTV-OCT software from our group. ${ }^{20}$ In short, the method uses a five-step algorithm to segment the images, identify individual particles, estimate the center-of-mass location of each particle, pair particles on sequential images, and estimate image-to-image displacement of the center-of-mass location of individual particles. Typical OCT imaging parameters were as follows: A-scan rate, $28 \mathrm{kHz}$; lateral imaging field of view, $3.5 \mathrm{~mm}$; A-scans per image, 2048; frame rate, $13.7 \mathrm{~Hz}$; images per acquisition session, 100. These typical parameters were occasionally adjusted to increase or decrease the frame rate in order to adequately capture particle displacements in samples that had notably faster or slower flow speeds, respectively.

Flow velocity summary statistics were generated by analyzing $\mathbf{v}(x, z)$ within a region of interest that was manually drawn within $\sim 100 \mu \mathrm{m}$ of the dorsal luminal surface. Our primary summary statistic was average flow speed. Average flow speed estimates were generated by analyzing histograms of $v_{x}(x, z)$ and $v_{z}(x, z)$. These histograms bin all measurements across $x$ and $z$ in the scalar component function over 100 frames $(6 \mathrm{~s}$ acquisition period). A representative raw histogram of $v_{x}(x, z)$ is shown in Fig. 1(b). Our scalar vector-component histograms typically showed a larger than expected number of counts centered at zero velocity. We attributed these peaks at zero velocity to (1) misidentification of portions of the stationary trachea as particles that are not moving and (2) particles that become stuck to the tracheal wall. In order to account for these artifacts, we replaced the zero-velocity histogram bin with the average of the two neighboring bins [Fig. 1(c)]. Our processing also addresses three sources of uncertainty that influence histogram width: (1) spatial heterogeneity of flow speeds over the region of interest, (2) Brownian motion, and (3) measurement uncertainty (e.g., uncertainty in the centroiding process used in particle tracking and failure of particle matching in-between frames). Since the latter two factors are expected to be zero-mean processes, vector component values within the region of interest, $v_{j, \text { avg }}$ were estimated using $v_{j, \text { avg }}=\sum_{i} c_{i j} n_{i j} / \sum_{i} n_{i j}$. Here, $j$ is the vector component direction $(x$ or $z), c_{i}$ is the center value of the bin, and $n_{i}$ is the number of counts in the $I$ 'th bin. In this formulation of $v_{j, \text { avg }}$, additive sources of zeromean noise should tend to zero. In contrast, calculating average speed values from $m$ individual particle velocities $\mathbf{v}_{m}\left(x_{m}, z_{m}\right)$ propagates the zero-mean noise forward when summing the squares of the vector components for each $m$ 'th particle (i.e., $\left.s_{m}^{2}=v_{x, m}^{2}+v_{z, m}^{2}\right)$. Finally, our summary statistic of average speed $s_{\text {avg }}$ was then estimated using $s_{\text {avg }}^{2}=v_{x, \text { avg }}^{2}+v_{z \text {,avg }}^{2}$.

As an initial validation of PTV-OCT to detect biomedically relevant flow perturbations in the sub- $100 \mu \mathrm{m} / \mathrm{s}$ regime, we quantified the temperature dependence of cilia-driven fluid flow velocity in mouse trachea. Average flow speeds were acquired from 12 mouse tracheae (Fig. 2). Consistent with prior literature, ${ }^{22}$ flow speeds increase as temperature increases from room to body temperature. Interestingly, after returning the temperature to a normal range of 36 to $38^{\circ} \mathrm{C}$ from febrile temperatures, flow velocities do not recover to baseline values for that temperature range. These results suggest that fever may impair 


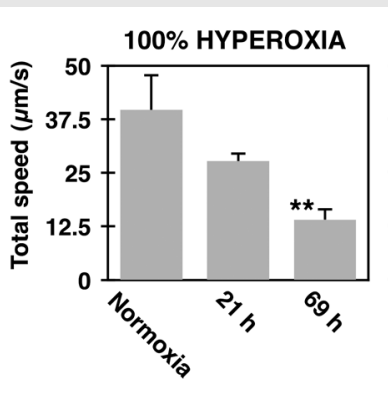

(a)

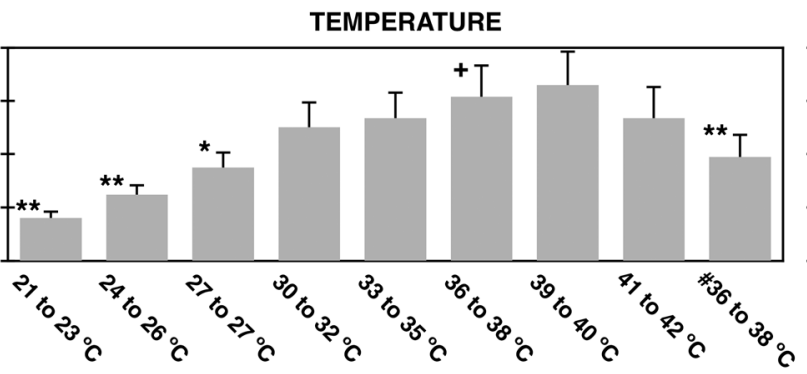

(b)

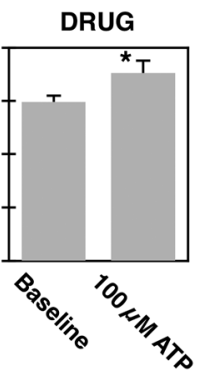

(c)

Fig. 2 (a) Exposure to hyperoxia (fraction of inspired oxygen $=100 \%$ ) for $69 \mathrm{~h}$ leads to damaged ciliadriven fluid flow performance. (b) Increasing temperature to body temperature (+) increased flow. Returning back to body temperature, indicated by $\# 36$ to $38^{\circ} \mathrm{C}$, after increasing to febrile temperatures does not restore baseline flow rates. (c) ATP increases cilia-driven fluid flow rates compared to baseline values. ${ }^{*}, p<0.05 ;{ }^{* *}, p<0.01$ [hyperoxia: Wilcoxon rank sum test (unpaired); temperature and drug: Wilcoxon signed rank test (paired)]. Error bars are standard error of the mean. $h$, hours; ATP, adenosine triphosphate.

flow performance even after fever has resolved. To provide additional validation of our PTV-OCT approach to detect biomedically relevant flow perturbations, we demonstrated pharmacological modulation of cilia-driven fluid flow in ex vivo adult mouse trachea. Exposure of adult tracheae (PND 42) to $100 \mu \mathrm{M}$ concentration of ATP for 10 to 15 min leads to a statistically significant increase in flow velocity $(n=10 ;$ Fig. 2$)$. Since OCT was not able to image through the thicker adult tracheal wall, the tracheal tube was opened to expose the ciliated epithelium for these experiments.

To investigate the effect of hyperoxia on cilia-driven fluid flow performance, groups of wild-type C57B6 PND 21 mice were exposed to hyperoxia $\left(\mathrm{FiO}_{2}=100 \%\right)$ for either $21 \mathrm{~h}$ $(n=5)$ or $69 \mathrm{~h}(n=13)$. Animals were exposed to hyperoxia by placing them in an airtight Plexiglas chamber with matched atmospheric pressure and regulated oxygen amount to $\mathrm{FiO}_{2}=$ $100 \%$. A control group of mice $(n=12)$ was kept in normoxic conditions. All mouse work was approved by the Institutional Animal Care and Use Committee at the Yale Medical School. Cilia-driven fluid flow was measured using PTV-OCT ex vivo in excised tracheae kept at 36 to $38^{\circ} \mathrm{C}$. There was a statistically significant difference between average flow speed between the $69 \mathrm{~h}$ hyperoxia group and the normoxic controls (39.7 versus $14 \mu \mathrm{m} / \mathrm{s}$, respectively; $p=0.01)$. There was no statistical significant difference between the $21 \mathrm{~h}$ hyperoxia and the normoxic controls (27.7 versus $14 \mu \mathrm{m} / \mathrm{s}$, respectively; $p=0.79$ ). Using separate groups of mice, tracheae from four normoxic and seven $69 \mathrm{~h}$ hyperoxia-exposed mice underwent hematoxolyn and eosin (H\&E) histopathological examination. There were subtle

Table 1 Summary of subtle histopathological findings in hyperoxiaexposed trachea.

Ciliated epithelial cells Mucin granules Goblet cells

\begin{tabular}{lccc}
\hline Hyperoxia \#1 & $\begin{array}{l}\text { Focal loss of cilia with } \\
\text { mildly abnormal cells }\end{array}$ & Mild increase & Normal \\
Hyperoxia \#2 & Normal & Mild increase & Normal \\
Hyperoxia \#3 & Normal & Mild increase & Mild increase \\
\hline
\end{tabular}

Note: Three of seven hyperoxia-exposed animals had abnormal findings by at least one criteria. abnormal findings on three of the seven hyperoxia-exposed animals (Table 1). Five features of the trachea were assessed: ciliated epithelial cells, mucin granules, goblet cells, submucosal space, and tracheal cartilage. Unless noted in Table 1, these features were normal in hyperoxia-exposed animals. One of the normoxic tracheae showed detachment of tracheal cells consistent with damage from tissue handing and preparation. Otherwise, the normoxic trachea appeared normal on H\&E examination. In all, $69 \mathrm{~h}$ of $100 \% \mathrm{FiO}_{2}$ in PND 21 mice led to (1) subtle abnormal findings in a minority of trachea examined using H\&E and (2) diminution but not elimination of ciliadriven fluid flow.

In sum, PTV-OCT imaging of ex vivo mouse tracheae is a powerful approach to quantitatively investigate novel factors that damage and modulate cilia-driven fluid flow in the sub-100 $\mu \mathrm{m} / \mathrm{s}$ regime (sub-0.25 in./min regime). We report the finding of hyperoxia-mediated damage to the capacity of a mammalian tracheal epithelium to generate fluid flows. We also report data suggesting flow impairment due to febrile temperatures may not reverse upon restoration of normal body temperature. We expect that the results presented here will lead to more detailed research into the mechanism and pharmacological treatment (e. g., ATP) of cilia-driven flow reduction caused by common clinical interventions and exposures.

\section{Acknowledgments}

This work was supported by March of Dimes Basil O'Connor Starter Scholar Research Award NIH1R01HL118419-01 (M.A.C.) and NIHR01HL085103 (V.B.). B.K.H. was additionally supported by NIH MSTP TG T32GM07205.

\section{References}

1. E. Maltepe and O. D. Saugstad, "Oxygen in health and disease: regulation of oxygen homeostasis-clinical implications," Pediatr. Res. 65(3), 261-268 (2009).

2. M. Hallman et al., "Better neonatal outcomes: oxygen, surfactant and drug delivery. Preface," Neonatology 103(4), 316-319 (2013).

3. D. L. Urso, "Treatment for acute asthma in the Emergency Department: practical aspects," Eur. Rev. Med. Pharmacol. Sci. 14(3), 209-214 (2010).

4. J. K. Stoller et al., "Oxygen therapy for patients with COPD: current evidence and the long-term oxygen treatment trial," Chest 138(1), 179-187 (2010). 


\section{JBO Letters}

5. V. Bhandari, "Hyperoxia-derived lung damage in preterm infants," Semin. Fetal Neonatal Med. 15(4), 223-229 (2010).

6. V. Bhandari, "Molecular mechanisms of hyperoxia-induced acute lung injury," Front. Biosci. 13, 6653-6661 (2008).

7. C. J. Wright and P. A. Dennery, "Manipulation of gene expression by oxygen: a primer from bedside to bench," Pediatr. Res. 66(1), 3-10 (2009).

8. P. A. Dennery, "Oxidative stress in development: nature or nurture?," Free Radic. Biol. Med. 49(7), 1147-1151 (2010).

9. A. Bhandari and V. Bhandari, "Pitfalls, problems, and progress in bronchopulmonary dysplasia," Pediatrics 123(6), 1562-1573 (2009).

10. E. Baraldi and M. Filippone, "Chronic lung disease after premature birth,” N. Engl. J. Med. 357(19), 1946-1955 (2007).

11. B. Weinberger et al., "Oxygen toxicity in premature infants," Toxicol. Appl. Pharmacol. 181(1), 60-67 (2002).

12. E. de Jonge et al., "Association between administered oxygen, arterial partial oxygen pressure and mortality in mechanically ventilated intensive care unit patients," Crit. Care 12(6), R156 (2008).

13. W. A. Altemeier and S. E. Sinclair, "Hyperoxia in the intensive care unit: why more is not always better," Curr. Opin. Crit. Care 13(1), 73-78 (2007)

14. B. J. Stenson and J. A. Orme, "The twists and turns of neonatal oxygen therapy," Early Hum. Dev. 88(12), 961-963 (2012).
15. V. Konradova et al., "Mild hyperoxia induces moderate pathological alteration in airway epithelium (ultrastructural study)," Vet. Med. (Czech). 48(11), 313-320 (2003).

16. M. B. Hershenson et al., "Hyperoxia increases airway cell S-phase traversal in immature rats in vivo," Am. J. Respir. Cell Mol. Biol. 11(3), 296-303 (1994).

17. M. B. Hershenson et al., "Hyperoxia-induced airway hyperresponsiveness remodeling in immature rats," Am. J. Physiol. 262(3), L263-L269 (1992).

18. M. A. Sackner et al., "Effect of oxygen in graded concentrations upon tracheal mucous velocity. A study in anesthetized dogs," Chest J. 69(2), 164-167 (1976).

19. S. Yuan et al., "Oxidation increases mucin polymer cross-links to stiffen airway mucus gels," Sci. Transl. Med. 7(276), 276 ra27 (2015).

20. S. Jonas et al., "Microfluidic characterization of cilia-driven fluid flow using optical coherence tomography-based particle tracking velocimetry," Biomed. Opt. Express 2(7), 2022-2034 (2011).

21. B. K. Huang et al., "Quantitative optical coherence tomography imaging of intermediate flow defect phenotypes in ciliary physiology and pathophysiology," J. Biomed. Opt. 20(3), 030502 (2015).

22. A. B. Christopher et al., "The effects of temperature and anesthetic agents on ciliary function in murine respiratory epithelia," Front. Pediatr. 2, 111 (2014). 